\title{
Criticality in failure under compression: Acoustic emission study of coal and charcoal with different microstructures
}

\author{
Yangyang Xu, ${ }^{1}$ Angeles G. Borrego, ${ }^{2}$ Antoni Planes, ${ }^{3,4}$ Xiangdong Ding, ${ }^{1}$ and Eduard Vives ${ }^{3,5}$ \\ ${ }^{1}$ State Key Laboratory for Mechanical Behavior of Materials, Xi'an Jiaotong University, Xi'an 710049, China \\ ${ }^{2}$ Instituto Nacional del Carbón-CSIC, Fracisco Pintado Fe, 26, 33011 Oviedo, Asturias, Spain \\ ${ }^{3}$ Departament de Física de la Matèria Condensada, Facultat de Física, Universitat de Barcelona, \\ Martí i Franquès 1, 08028 Barcelona, Catalonia, Spain \\ ${ }^{4}$ Institute of Nanoscience and Nanotechnology (IN2UB), Barcelona, Catalonia, Spain \\ ${ }^{5}$ Universitat de Barcelona Institute of Complex Systems (UBICS), Barcelona, Catalonia, Spain
}

(Received 31 October 2018; published 4 March 2019)

\begin{abstract}
A systematic study of acoustic emission avalanches in coal and charcoal samples under slow uniaxial compression is presented. The samples exhibit a range of organic composition in terms of chemical elements as well as different degrees of heterogeneity in the microstructure. The experimental analysis focuses on the energies $E$ of the individual acoustic emission events as well as on the time correlations between successive events. The studied samples can be classified into three groups. The more homogeneous samples (group I) with pores in the micro and nanoscales, with signatures of hardening effects in the stress-strain curves, exhibit the cleanest critical power-law behavior for the energy distributions $g(E) d E \sim E^{-\epsilon} d E$ with a critical exponent $\epsilon=1.4$. The more heterogeneous samples with voids, macropores, and granular microstructures (group III), show signatures of weakening effects and a larger effective exponent close to the value $\epsilon=1.66$, but in some cases truncated by exponential damping factors. The rest of the samples (group II) exhibit a mixed crossover behavior still compatible with an effective exponent $\epsilon=1.4$ but clearly truncated by exponential factors. These results suggest the existence of two possible universality classes in the failure of porous materials under compression: one for homogeneous samples and another for highly heterogeneous samples. Concerning time correlations between avalanches, all samples exhibit very similar waiting time distributions although some differences for the Omori aftershock distributions cannot be discarded.
\end{abstract}

DOI: 10.1103/PhysRevE.99.033001

\section{INTRODUCTION}

The study of the intermittent response of heterogeneous materials under smooth external driving is important from both fundamental and applied points of view. Stochastic responses consisting in a series of avalanche events (also called crackling noise [1]) have been found associated with very different phenomena: structural transitions in solids [2], fracture [3], magnetization processes in ferromagnets [4], polarization in ferroelectrics [5], superconductors [6], condensation [7], and so on. A deep understanding of such phenomena can be very important for the prediction of catastrophic events like earthquakes, natural and man-made structure collapses, bone breaking, and so on.

Five years ago, acoustic emission (AE) experiments [8] revealed that some porous materials under uniaxial compression show features compatible with the existence of an outof-equilibrium critical point. Under slow mechanical driving, the amplitudes and energies of the successive avalanche events exhibit power-law distributions extending five and nine decades, respectively [9], thus clearly revealing a lack a characteristic scales. Moreover it was evidenced that the instants of failure events are non-Poissonian. They exhibit temporal clustering correlations which also show critical properties. The rate of aftershocks after big events decays with time as an Omori power law and the distribution of waiting times is characterized by a universal double power-law distribution.
In this context, different porous materials have been studied in similar conditions. By far, the cleanest experimental evidences of criticality [9] are shown by Vycor samples, a mesoporous quartz $\left(\mathrm{SiO}_{2}\right.$, with pore diameters in the range $2-10 \mathrm{~nm}$ ) synthetized by a phase separation and leaching process. Qualitatively similar features have been found in other synthetic $\mathrm{SiO}_{2}$ materials (Gelsil) [10], synthetic porous alumina $\left(\mathrm{Al}_{2} \mathrm{O}_{3}\right)$ [11], porous berlinite $\left(\mathrm{AlPO}_{4}\right)$ [12], natural minerals like Goethite (FeO-OH) [13], rocks like sandstone [10] or shale [14], and biological samples like wood [15] and bones [16]. It should be mentioned that some previous works have studied AE avalanches in coal or charcoal but without much emphasis on the influence of the microstructure: critical behavior similar to earthquakes was found in ethanoldampened charcoal [17], and unspecified coal samples were studied under compression for the analysis of record-breaking acoustic emission signals [18].

Despite the numerous experimental efforts, at present, it is not clear what are the physico-chemical factors that favor the existence of a clean critical behavior extending several decades or, contrarily, introduce noncritical exponential damping factors to the power-law distributions. The different works have tried to assess what is the influence of the driving rate [8], the driving mechanism [19], sample porosity [11], granularity [20], chemical bonding [10,13,14], existence of impurities [12], and so on without fully conclusive results. 
There is also an open debate on whether the behavior can be described as an approach to a single critical point or to a stationary critical behavior [21]. Moreover it would be interesting to elucidate whether or not there exist "universality" classes characterized by different sets of critical exponents.

The final goal of this paper is to contribute to clarify this scenario by a systematic study of charcoal and natural coal samples exhibiting a rich variety of microstructures from more homogeneous to highly heterogeneous.

In Sec. II we detail the statistical methods that will be used for the study of the acoustic emission avalanches. In Sec. III we specify the characteristic of the charcoal and the natural coal samples. In Sec. IV we describe the experimental setup. In Sec. V we present our results, that are discussed and compared to previous data from the literature in Sec. VI. Finally, in Sec. VII we summarize and conclude.

\section{METHODS FOR AVALANCHE ANALYSIS}

The sequences of ultrasonic AE avalanches obtained by compressing porous materials (often called "labquakes") share with natural earthquakes very similar statistical laws governing its properties. Natural seisms can easily be studied from public available catalogues corresponding to individual faults, large regions on earth or even to the full globe. They extend in time for many years or even decades and include all the registered events above a certain energy threshold.

Energy distributions of earthquakes exhibit the so-called Gutenberg-Richter law. Although it is usually formulated in terms of cumulative distribution of earthquake magnitudes it can be translated into a power-law probability density $g(E)$ for the earthquake energy (seismic moment [22]). The probability of finding an earthquake with energy between $E$ and $E+d E$ is given by

$$
d P(E)=g(E) d E=\left(\frac{\epsilon-1}{E_{\min }^{1-\epsilon}}\right) E^{-\epsilon} d E,
$$

where $\epsilon$ is the so-called Gutenberg-Richter exponent (usually taking values close to $\epsilon=5 / 3$ [23]), and the prefactor within parentheses is the normalization constant that ensures that the probability density is well normalized between an unavoidable minimum cutoff $E_{\min }$ and $\infty$. (The relation of the $\epsilon$ exponent with the exponent $b$ commonly used in seismology is $\epsilon=1+\frac{2}{3} b$ assuming that the relation between the magnitude $M$ and the energy $E$ follows $M \propto \frac{2}{3} \log _{10} E$ [22]. Therefore $\epsilon=5 / 3 \simeq 1.66$ is equivalent to $b=1$.)

Fitting the exponent $\epsilon$ of a power-law probability density with a good accuracy requires the use of techniques that are independent of the histogram representation. This can easily be achieved by maximum likelihood (ML) methods [24,25]. Moreover, when the ML fit is restricted to energies recorded above a given lower cutoff $E_{\text {low }}$ one expects that the estimator of the exponent will be constant when $E_{\text {low }}$ is changed for several decades [26]. If this is not the case, and the fitted exponent shows a systematic increase with $E_{\text {low }}$ it is usually indicative of the existence of a exponential damping factor in the probability distribution revealing the existence of a characteristic energy $\Lambda_{E}$, i.e.,

$$
d P(E)=g(E) d E \propto E^{-\epsilon} e^{-E / \Lambda_{E}} d E .
$$

As already mentioned, other important statistical properties of natural earthquakes are related to its non-Poissonian behavior. Possible future forecast methods rely on a deep understanding of such temporal correlations. They can be revealed by studying, for instance, the so-called sequences of aftershocks (AS), discovered by Omori [27] more than one century ago. After a large earthquake (called mainshock, MS) the seismic activity shows a clear increase that decays with time like a power law. The discovery of this law was possible through the study of a single large earthquake by recording the number of events in a relatively small region close to the original seism for a very long time. The analysis is more problematic when one needs to extend it to a full catalog of events in a certain large region [28]. Quantitative results might depend on the exact definition of MS and AS. A standard method consists of defining as MS all the events with energies within a certain window $E_{\mathrm{MS}} \pm \Delta E$ (with $E_{\mathrm{MS}}$ typically high). After each MS, the sequence of recorded events with energies smaller than the MS are assumed to be AS, generated by the MS. The AS sequence ends as soon as an event larger than the parent MS is found. By analyzing a large number of AS sequences one can study the behavior of the average AS rate $r_{\mathrm{AS}}$ (average number of AS per unit time) as a function of the time distance from the $\mathrm{MS}$ ( $\left.\Delta t=t-t_{\mathrm{MS}}\right)$. This reveals a power-law decay:

$$
r_{\mathrm{AS}}(\Delta t)=\frac{K}{(c+\Delta t)^{p}},
$$

where $K$ is a constant independent of time, $p$ is the Omori exponent, and $c$ is a tiny shift that avoids a divergence at $\Delta t=0$ and is related to the fact that very close to the MS, there is usually an undercounting of AS (overlapped with the MS). On earthquake catalogues, that contain epicenter information, one also requires that AS should occur within a certain distance from the MS. In labquakes, such spatial information is usually not available and thus this restriction is not imposed, and one assumes that the samples are small enough so that all the events smaller and subsequent to the MS are causally related to the MS.

If earthquakes were Poissonian, the rate of aftershocks in Eq. (3) would be constant and independent of the time to the MS $(p=0)$. Contrarily, the exponent $p$ shows a value typically close to $p \simeq 1$. Moreover, the constant $K$ in Eq. (3) that determines the total number of AS after a mainshock, depends on the energy of the MS also through a power-law function. This is the so-called Productivity law [28]

$$
K=K_{0} E_{\mathrm{MS}}^{\frac{2}{3} \alpha}
$$

with $K_{0}$ being a constant and the exponent $\alpha$ taking values $\alpha \simeq 0.8$. If the exponents $\alpha$ and $p$ are universal, the combination of Eqs. (3) and (4) allows to perform a scaling plot by representing $E_{\mathrm{MS}}^{-2 / 3 \alpha} r_{\mathrm{AS}}$ versus $\Delta t=t-t_{\mathrm{MS}}$ in a log-log plot

$$
E_{\mathrm{MS}}^{-\frac{2}{3} \alpha} r_{\mathrm{AS}}=K_{0}(c+\Delta t)^{-p} \text {. }
$$

The resulting curve, with a slope $-p$ (in log-log plot) should be independent of (i) the window $\Delta E$ used for the definition of the MS, (ii) the studied region, and (iii) the time window of the catalog.

Independently of the behavior described above, the nonPoissonian character of the event sequence can also be 
TABLE I. List of coal and charcoal samples studied in this work. The first nine columns show the chemical parameters and the rest the petrographic parameters. The reflectance of vitrinite [column 16, VitR $(\%)$ ] is a good indicator of rank and is used to order the samples in the table $(\mathrm{d}=$ dry basis, $\mathrm{a}=$ dry-ash-free basis, $*=$ atomic ratio, vol $=$ volume, $\mathrm{s}=$ standard deviation, Rscan $=$ maceral reflectance ponderate).

\begin{tabular}{|c|c|c|c|c|c|c|c|c|c|c|c|c|c|c|c|c|c|c|c|c|}
\hline $\begin{array}{l}\text { Sample } \\
\text { Name }\end{array}$ & $\begin{array}{l}\text { Ash } \\
(\mathrm{d} \%)\end{array}$ & $\begin{array}{c}\text { Volatile } \\
(\mathrm{a} \%)\end{array}$ & $\begin{array}{c}\mathrm{C} \\
(\mathrm{a} \%)\end{array}$ & $\begin{array}{c}\mathrm{H} \\
(\mathrm{a} \%)\end{array}$ & $\begin{array}{c}\mathrm{N} \\
(\mathrm{a} \%)\end{array}$ & $\begin{array}{c}\mathrm{St} \\
(\mathrm{a} \%)\end{array}$ & $\begin{array}{c}\mathrm{O} \\
(\mathrm{a} \%)\end{array}$ & $\begin{array}{c}\mathrm{O} / \mathrm{C} \\
(*)\end{array}$ & $\begin{array}{c}\mathrm{H} / \mathrm{C} \\
(*)\end{array}$ & $\begin{array}{c}\mathrm{Rr} \\
(\mathrm{d} \%)\end{array}$ & $\begin{array}{c}\text { Vit } \\
(\operatorname{vol} \%)\end{array}$ & $\begin{array}{c}\text { Int } \\
(\operatorname{vol} \%)\end{array}$ & $\begin{array}{c}\text { Lip } \\
\text { (vol \%) }\end{array}$ & $\begin{array}{c}\text { Rscan } \\
(\%)\end{array}$ & $\begin{array}{l}\text { VitR } \\
(\%)\end{array}$ & $\mathrm{s}$ & $\begin{array}{l}\text { IntR } \\
(\%)\end{array}$ & $\mathrm{s}$ & $\begin{array}{c}\text { LipR } \\
(\%)\end{array}$ & $\mathrm{s}$ \\
\hline Charcoal & 0.8 & 38.1 & 77.6 & 3.8 & 0.4 & 0 & 18.3 & 0.18 & 0.58 & & & & & & & & 0.97 & 0.13 & & \\
\hline FG1 & 11.3 & 40.7 & 71.8 & 4.3 & 1.6 & 0.6 & 21.7 & 0.23 & 0.72 & 0.49 & 57.1 & 39.4 & 3.5 & 0.75 & 0.48 & 0.06 & 1.26 & 0.46 & 0.13 & 0.07 \\
\hline VLD & 8.8 & 39.3 & 74.2 & 4.8 & 0.6 & 0.2 & 20.2 & 0.20 & 0.78 & 0.56 & 64.7 & 29.9 & 5.4 & 0.73 & 0.53 & 0.05 & 1.27 & 0.45 & 0.14 & 0.11 \\
\hline INS & 1.6 & 46.0 & 78.2 & 5.4 & 1.6 & 0.5 & 14.3 & 0.14 & 0.83 & 0.58 & 91.8 & 4.0 & 4.2 & 0.55 & 0.56 & 0.06 & 0.84 & 0.23 & 0.15 & 0.09 \\
\hline $\mathrm{COS}$ & 1.2 & 42.9 & 79.9 & 5.4 & 1.5 & 0.5 & 12.7 & 0.12 & 0.81 & 0.60 & 84.4 & 13.2 & 2.4 & 0.66 & 0.58 & 0.05 & 1.26 & 0.43 & 0.13 & 0.1 \\
\hline WA2 & 6.6 & 36.9 & 80.8 & 5.1 & 1.9 & 0.3 & 11.9 & 0.11 & 0.76 & 0.66 & 57.2 & 37.3 & 5.5 & 0.92 & 0.64 & 0.04 & 1.38 & 0.58 & 0.21 & 0.08 \\
\hline $\mathrm{LOH}$ & 10.8 & 35.8 & 81.8 & 5.0 & 1.7 & 0.4 & 11.1 & 0.10 & 0.73 & 0.79 & 85.2 & 9.0 & 5.8 & 0.81 & 0.79 & 0.05 & 1.35 & 0.34 & 0.13 & 0.03 \\
\hline PHA & 15.3 & 36.7 & 81.3 & 5.2 & 1.3 & 2.6 & 9.6 & 0.09 & 0.77 & 0.84 & 84.3 & 9.1 & 6.6 & 0.84 & 0.87 & & 2.01 & & 0.23 & \\
\hline CRB & 7.1 & 28.6 & 87.4 & 4.9 & 2.0 & 0.2 & 5.5 & 0.05 & 0.67 & 0.97 & 57.2 & 42.2 & 0.6 & 1.21 & 0.95 & 0.06 & 1.63 & 0.38 & 0.52 & 0.17 \\
\hline SMK & 12.8 & 18.1 & 90.6 & 4.4 & 1.1 & 0.4 & 3.5 & 0.03 & 0.58 & 1.53 & 61.6 & 38.4 & & 1.77 & 1.54 & 0.08 & 2.08 & 0.44 & & \\
\hline DAN & 2.3 & 6.2 & 92.1 & 3.2 & 1.7 & 0.5 & 2.5 & 0.02 & 0.42 & 3.20 & 61.8 & 38.2 & & 3.22 & 3.18 & 0.26 & 3.28 & 0.46 & & \\
\hline VCB & 1.9 & 1.6 & 94.9 & 1.2 & 0.9 & 0.2 & 2.8 & 0.02 & 0.15 & 5.63 & 98.0 & 2.0 & & 5.63 & 5.63 & 0.35 & 5.67 & 0 & & \\
\hline VAN & 3.0 & 1.9 & 94.2 & 1.4 & 0.9 & 0.3 & 3.2 & 0.03 & 0.17 & 5.76 & 98.4 & 1.6 & & 5.75 & 5.78 & 0.21 & 5.92 & 0.11 & & \\
\hline
\end{tabular}

revealed by the study of the waiting times $\delta$ between consecutive earthquakes with energies above a certain threshold $E_{t h}$. The variation of this additional threshold (besides the experimental minimum threshold determined by instruments sensitivity) allows to study, from a unique recorded catalogue in a certain region, the behavior of waiting times in sparse (high $E_{t h}$ ) or more dense (low $E_{t h}$ ) sequences, similarly to what would correspond to the behavior in regions with low or high activity.

If the studied catalogues are large enough and include regions and/or periods of time that clearly exhibit activity rates spanning several orders of magnitude, the distribution of waiting times $g(\delta) d \delta$ obeys a universal scaling law (USL) with a double power-law shape $[8,29]$ given by

$$
g(\delta) d \delta=\Phi(\delta /\langle\delta\rangle) \frac{d \delta}{\langle\delta\rangle},
$$

where $\langle\delta\rangle$ is the average waiting time of the catalog (that typically depends on the energy threshold $E_{t h}$, the region, the time window, etc.) and $\Phi$ is a universal function

$$
\Phi(x) \propto \begin{cases}x^{-(1-v)} & \text { if } x \ll 1, \\ x^{-(2+\xi)} & \text { if } x \gg 1,\end{cases}
$$

where the exponents take values $(1-v) \simeq 0.9$ and $(2+\xi) \simeq$ 2.2 [29]. The relation between $(1-v)$ and $p$ exponents has been extensively discussed [30,32].

The goal of this work is to test these three laws (1), (5), and (6) for labquakes in a large set of coal and charcoal samples and conclude about the existence or not of different sets of exponent values that would be indicative of the existence of different universality classes.

\section{SAMPLES}

Coal is essentially constituted of a reduced number of chemical elements $(C, N, O, H$, and $S)$ that made part of former biological entities which have been subjected to complex geological histories. The combination of (i) different proportions of vegetal debris with different functions in the plant, (ii) early transformation conditions able to preserve the organic matter, and (iii) geothermal history linked to burial, creates a variety of structures within a relatively narrow range of chemical composition. In contrast to minerals, coal does not have a crystalline structure neither a fixed chemical composition since the composition of the various organic components (macerals) varies with thermal evolution [31]. The spatial arrangement of the organic elements generates also a complexity in the porosity and pore size distribution, within the macerals and within the coals as a whole.

Historically, coal has been classified in four classes: lignite, subbituminous, bituminous, and anthracite. These have been mostly established using utilization parameters such as calorific value and volatile matter yield, with boundaries between classes varying from country to country. A widely applicable classification would require to take into account three variables: grade, type, and rank (ISO 11760:2005). Grade refers to the inorganics or minerals amount, determined essentially as ash yield after high temperature treatment of the samples. Type relates to the proportion of the different organic components or macerals; and rank relates to the level of maturity reached during the burial process, determined by the time, temperature, and pressure.

Table I shows the list of coal samples studied in this work and some of its physical and chemical properties. The samples, belonging to the collection of INCAR (Instituto Nacional del Carbon, Spain), have been collected as single seam coals in different mines around the world and preserved from alteration. The parameters provided in Table I are those relevant for the assessment of coal grade (ash yield weight $\%$ dry basis, ISO 1171:2010), type (maceral composition in volume \% determined by optical microscopy through point counting, ISO 7404-03:2009), and rank (vitrinite reflectance as percentage of reflected light compared to a standard ISO 7404-05:2009, volatile matter yield, ISO 17246:2010 and elemental composition expressed as weight \% on a dry-ashfree basis as in ISO 29541:2010 and ISO 19579:2006), which are considered key parameters for coal characterization. The ash yield reflects the mineral matter incorporated as impurities to the coal. The maceral group composition reflects the relative proportion of plant components derived from: 


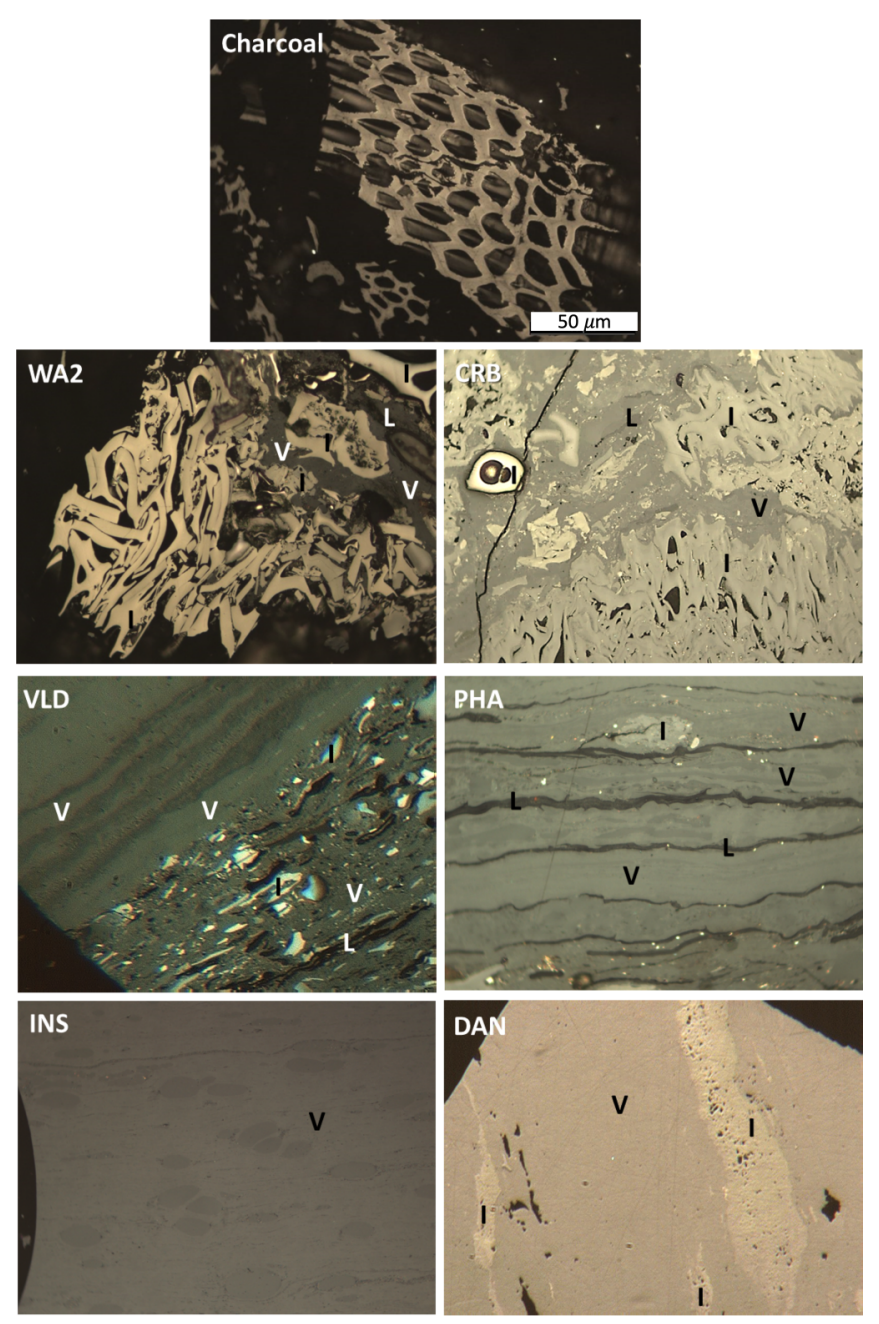

FIG. 1. Optical microscope images taken on reflected light oil immersion, revealing the microstructure of seven selected samples. Macerals are indicated by letters: V (vitrinite), I (inertinite), L (liptinite). The scale of the seven images is the same and it is indicated in the charcoal micrograph.

(i) lignocellulosic tissues having undergone humification prior to significant burial (vitrinite); (ii) lignocellulosic tissues having undergone dehydration and devolatilization prior to burial including charcoalification as occurs in forest fires (inertinite); and (iii) lipidic-rich substances found in algae, and protective or reproductive organs of plants (liptinite) [33]. Most of the coals worldwide are rich in vitrinite although coals with moderate to high inertinite contents are not rare [34]. The coals selected in this work range, in rank from subbituminous to anthracite, in ash yield from very low to moderate, and in maceral composition from vitrinite-rich to moderately high in inertinite trying to have representatives of each type at the various rank intervals (ISO 11760 :2005). The transformations of coal with rank can be monitored by an increase in vitrinite reflectance and carbon content and a decrease in volatile matter yield, $\mathrm{H}$, and $\mathrm{O}$ contents [35]. For any given rank the volatile matter yield is the highest for liptinite and the lowest for inertinite [36] whereas the reflectance is the highest for inertinite and the lowest for liptinite [31].

We also studied (see first line in Table I) charcoal samples. These correspond to commercial available fine art fusains (HB
$5 \mathrm{~mm}$, NITRAM, Canada). Charcoal formed from wood by mild heat-treatment under nonoxidizing atmosphere would be from a genetic point of view similar to inertinite, although in charcoal the reflectance variation would be moderate due to controlled conditions and the cell-cavities remain open since the coalification has not left any footprint [37].

Figure 1 shows selected examples of the microstructures of coal and the comparison to charcoal. The letters (I), (L), and (V) indicate inertinite, liptinite, and vitrinite macerals, respectively. Note the similar structure of charcoal and the inertinite macerals in the other samples except for the fact that cell cavities are deformed and broken in inertinite whereas they remain quite intact in the charcoal. The sequence of images $\mathrm{WA} 2 \rightarrow \mathrm{CRB} \rightarrow \mathrm{VLD} \rightarrow \mathrm{PHA} \rightarrow \mathrm{INS} \rightarrow \mathrm{DAN}$ shows, qualitatively, how the structure varies from a very heterogeneous structure with voids and mixture of components with clearly different physical properties to a much more homogeneous structure. Note that this sequence does not fully correspond to the order (by rank) in Table I since some low rank samples like INS clearly are quite homogeneous despite the differences existing between macerals at low rank. This is because such samples are already more homogeneous in the original maceral composition (mostly vitrinite).

Concerning porosity, although it cannot be fully observed in the images, in general it is well established that it decreases with increasing coal rank as a consequence of overburden and volatiles release although for coals with over $90 \%$ of $\mathrm{C}$ the porosity increases again. Overal, macropores (with a diameter $\Phi>50 \mathrm{~nm}$ ) decrease with increasing rank and the opposite occurs with micropores $(\Phi<2 \mathrm{~nm})$, whereas mesopores ( $2 \mathrm{~nm}<\Phi<50 \mathrm{~nm}$ ) are only present in the medium rank coals [38].

\section{EXPERIMENTAL SETUP}

From the collected coal samples as well as from the commercial charcoal fusains, we have cut (with a diamond saw) parallelepipedic specimens with approximate square basis. The variability of the studied specimens is large. Coal samples have lateral size $8 \pm 1 \mathrm{~mm}$, transversal section $63 \pm 15 \mathrm{~mm}^{2}$, and height $14 \pm 2 \mathrm{~mm}$. Charcoal samples have lateral size $6.1 \pm 0.3 \mathrm{~mm}$, transversal section $37 \pm 4 \mathrm{~mm}^{2}$, and heigth $11 \pm 1 \mathrm{~mm}$. Special care has been taken to ensure that, after mild polishing, the upper and lower faces are parallel. Repeatability of the results has been tested by comparing at least two, but in some cases eight, specimens cut from each coal sample. Coal samples have an average density of $1.3 \pm 0.3 \mathrm{~g} / \mathrm{cm}^{3}$ and charcoal samples $0.33 \pm 0.03 \mathrm{~g} / \mathrm{cm}^{3}$.

The specimens have been compressed between two aluminium plates, driven at a constant speed of $0.01 \mathrm{~mm} / \mathrm{min}$ by a Zwick/Roell testing machine with electronic speed control. For the case of charcoal specimens, analysis at different compression rates $(0.1 \mathrm{~mm} / \mathrm{min}, 0.05 \mathrm{~mm} / \mathrm{min}, 0.01 \mathrm{~mm} / \mathrm{min}$, and $0.005 \mathrm{~mm} / \mathrm{min}$ ) have been performed. The compression plates contain embedded piezoelectric transducers with 9.5$\mathrm{mm}$ diameter, centered on the sample, at 2-mm distance from it. The good ultrasonic contact between the transducers and the plates, as well as between the plates and the sample is ensured by a thin vaseline film. 


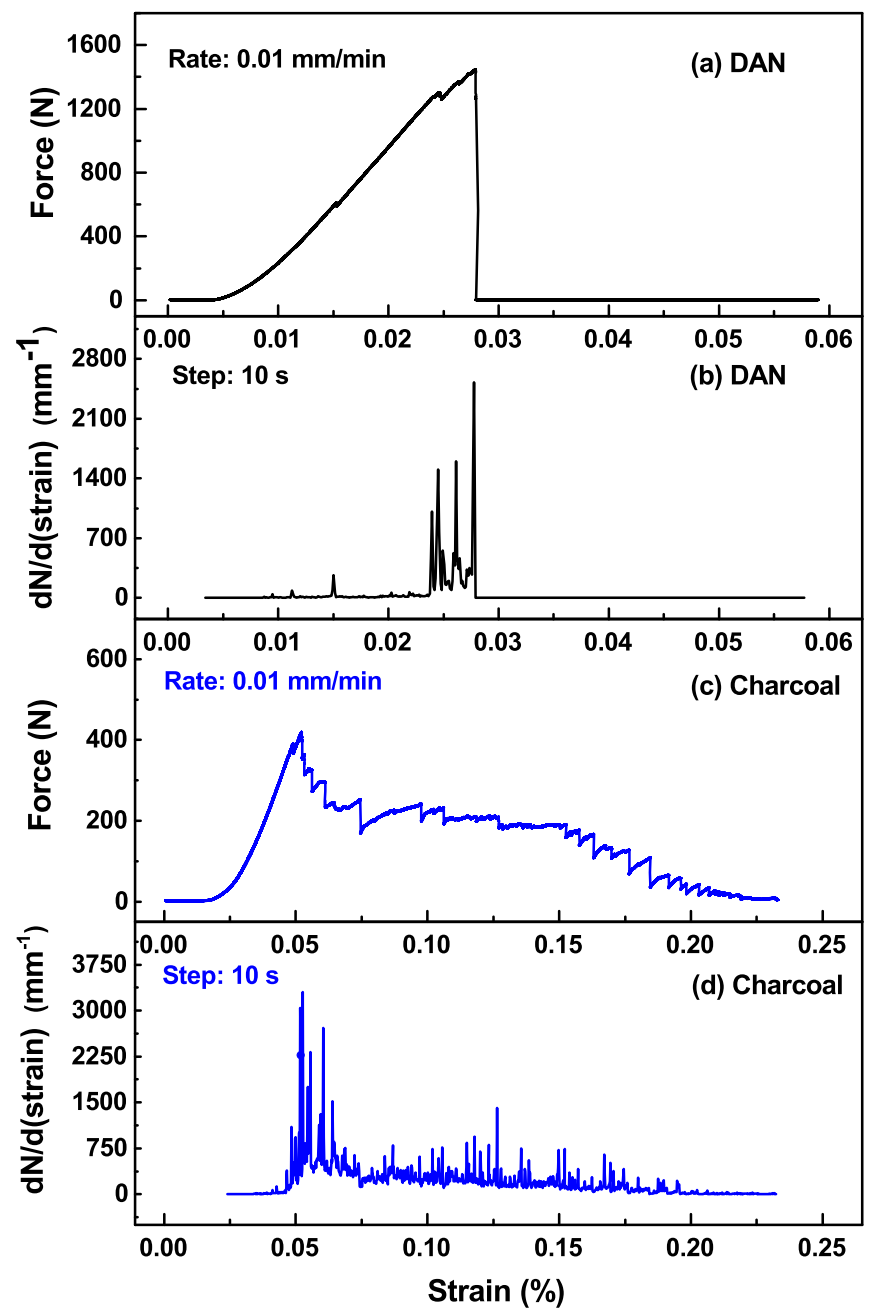

FIG. 2. Two examples of experimental data recorded in the present work, corresponding to specimens of DAN and Charcoa samples. Panels (a) and (c) correspond to the force versus strain behavior and panels (b) and (d) to the AE activity, displayed as the number of avalanche events every $10 \mathrm{~s}$.

The voltage signals detected by the transducers are first preamplified $(60 \mathrm{~dB})$ and sent to two separate channels, of a PCI2 system from Europhysical Acoustics. Individual AE events are defined or separated by using a threshold at $23 \mathrm{~dB}$. The threshold is selected as low as possible, but avoiding the detection of noise signals when the sample is not being compressed.

Events start when the voltage signal crosses the threshold, and finish when the voltage remains below threshold for more than $200 \mu \mathrm{s}$. The energy of the events is measured as the time integral of the square voltage during the whole event, divided by a reference resistance of $10 \mathrm{k} \Omega$. In each experiment we selected the signals only from the channel that recorded a larger number of events, thus indicating a better acoustic contact with the sample, and avoiding double counting of large signals that are simultaneously recorded by the two transducers.

Figure 2 shows two examples of the experimental data recorded by the setup corresponding to specimens of samples DAN and Charcoal. Figures 2(a) and 2(c) display the behavior of the force exerted by the compression plates as a function of
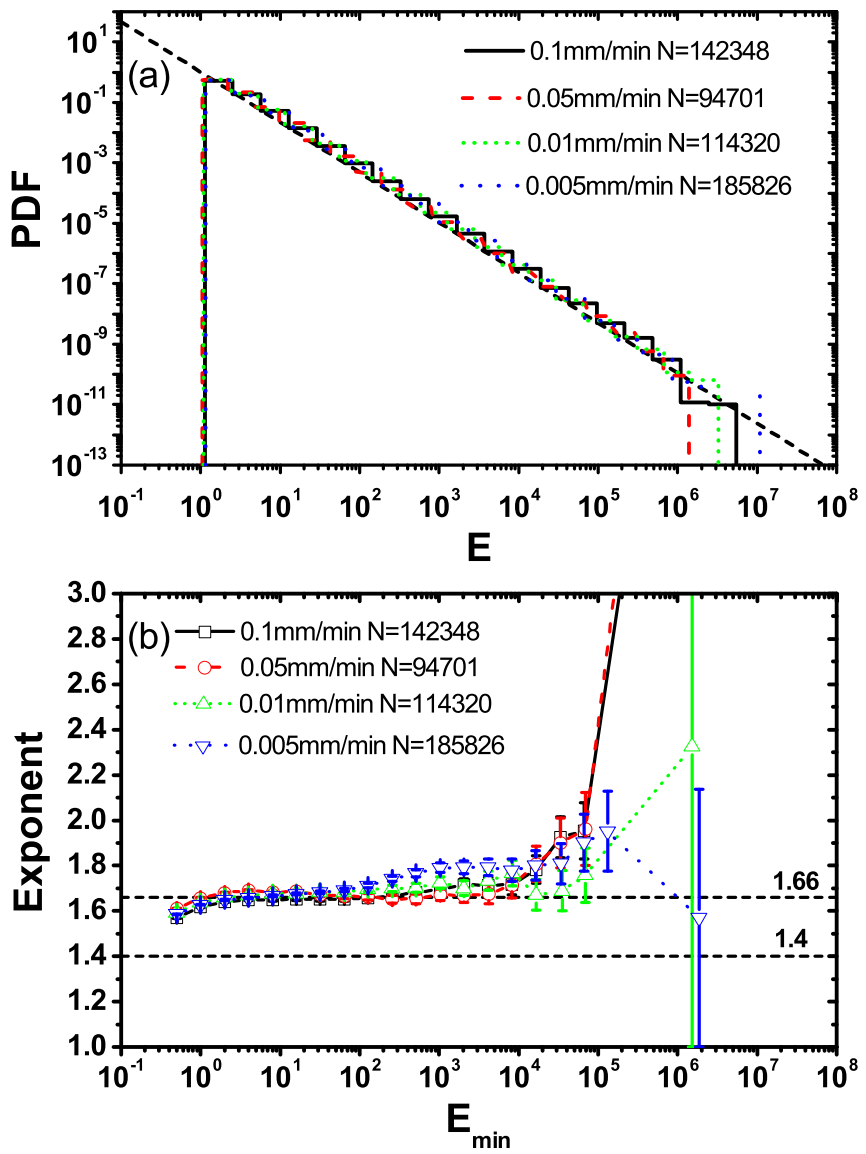

FIG. 3. (a) Histogram showing the energy distribution (in a log$\log$ plot, with logarithmic bins) for charcoal samples, at different compression rates as indicated by the legend. The values $N$ correspond to the number of events detected in each experiment. The dashed line corresponds to the power-law probability density with $\epsilon=1$.66. (b) Maximum likelihood estimation of the exponent $\epsilon$ as a function of a moving minimun energy cutoff. Dashed lines indicate the values $\epsilon=1.66$ and $\epsilon=1.4$.

strain (which is proportional to time given the constant compression velocity). Figures 2(b) and 2(d) display the behavior of the AE activity, shown as histograms corresponding to the number of recorded $\mathrm{AE}$ avalanche events every $10 \mathrm{~s}$.

Shortly after an initial adaptation regime (that is discarded from the analysis), the samples display an elastic regime with a monotonous increase of the stress as a function of the strain. In most cases, already for strains below 0.01 , AE events occur due to nucleation and growth of microfractures in the sample. The samples then typically enter in a serrated stress strain curve that reaches a maximum stress (yield point), after which the collapse occurs. In some cases [Fig. 2(a)] the collapse is rather sharp but in others [Fig. 2(c)] there are a series of subsequent collapses that might extend up to strains above 0.1 . The compression experiments end either when the force decreases below a prefixed low value $(5 \mathrm{~N})$ or when the strain approximately exceeds 0.2 and the activity has almost vanished.

\section{RESULTS}

A first set of experiments was performed with a large number of charcoal specimens to test the dependence of the results 

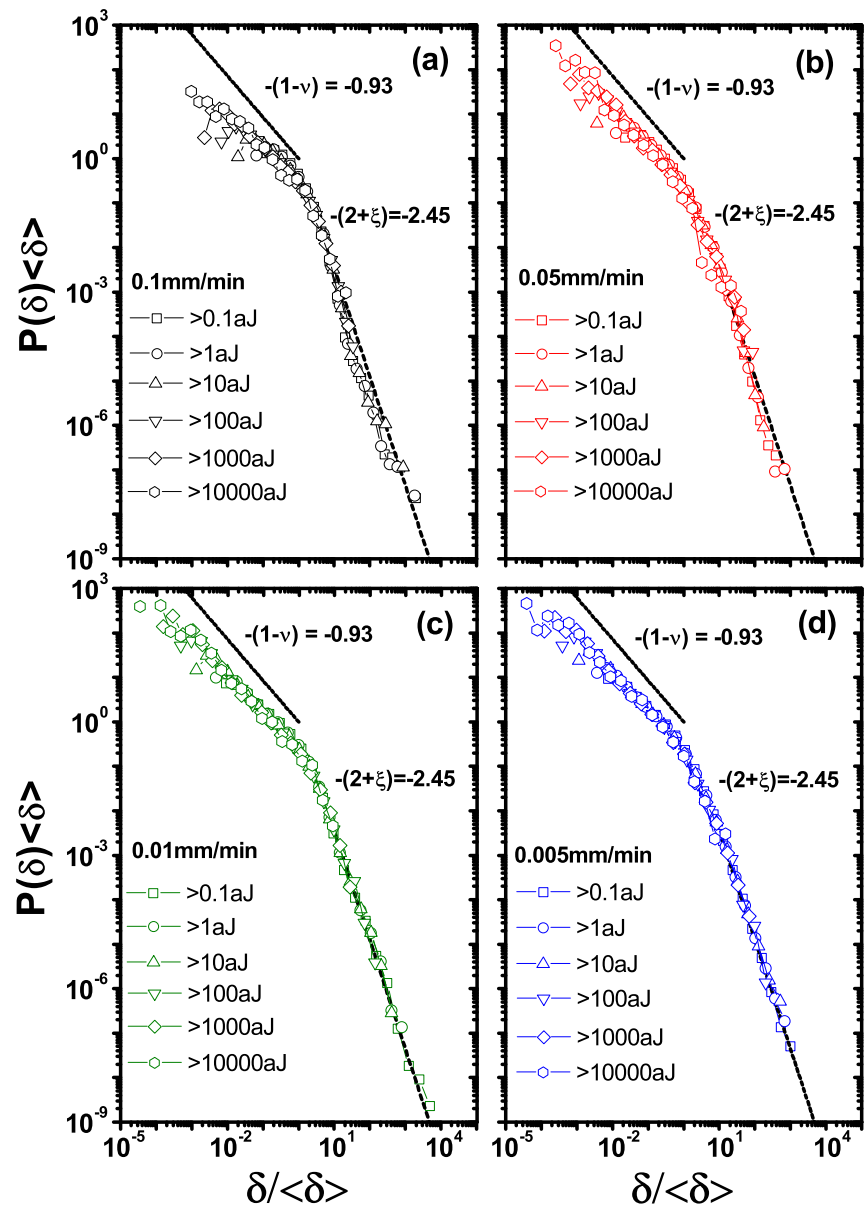

FIG. 4. Analysis of the waiting time probability distribution for charcoal samples at different compression rates as indicated by the labels. Data are shown in a log-log plot, scaled by the average waiting time $\langle\delta\rangle$. Different symbols correspond to different threshold levels $E_{t h}$ used for the estimation of the waiting times, as explained in Sec. II. The lines show the exponents found for Vycor in Ref. [8].

with the compression rate. Figure 3(a) shows the histograms revealing the distribution of energies for different compression rates from 0.05 to $0.1 \mathrm{~mm} / \mathrm{min}$. Below, in Fig. 3(b), we show the analysis of the corresponding Gutenberg-Richter exponent $\epsilon$, fitted by the ML method, as a function of a moving lower cutoff $E_{\min }$. As can be seen, the fitted exponent is rather constant for five decades with a value compatible with $\epsilon=1.66$, similar to the value $5 / 3$ found in real earthquakes. For comparison a dashed line with the exponent $\epsilon=1.4$ found previously for Vycor [8] is also shown. The compression rate should be slow enough to avoid overlapping of consecutive events, thus maximizing the number of recorded events. But, if the compression rate is too slow, the experiments last longer and then one might include more signals corresponding to external noise or thermally activated effects [39]. As can be seen, the rate $0.01 \mathrm{~mm} / \mathrm{min}$ seems to render the best plateau (five decades) and a reasonable large number of recorded signals.

The influence of the compression rate on the time correlation between events is also tested for charcoal samples by plotting the distribution of waiting times defined for different energy thresholds $E_{t h}$. Figure 4 shows the universal
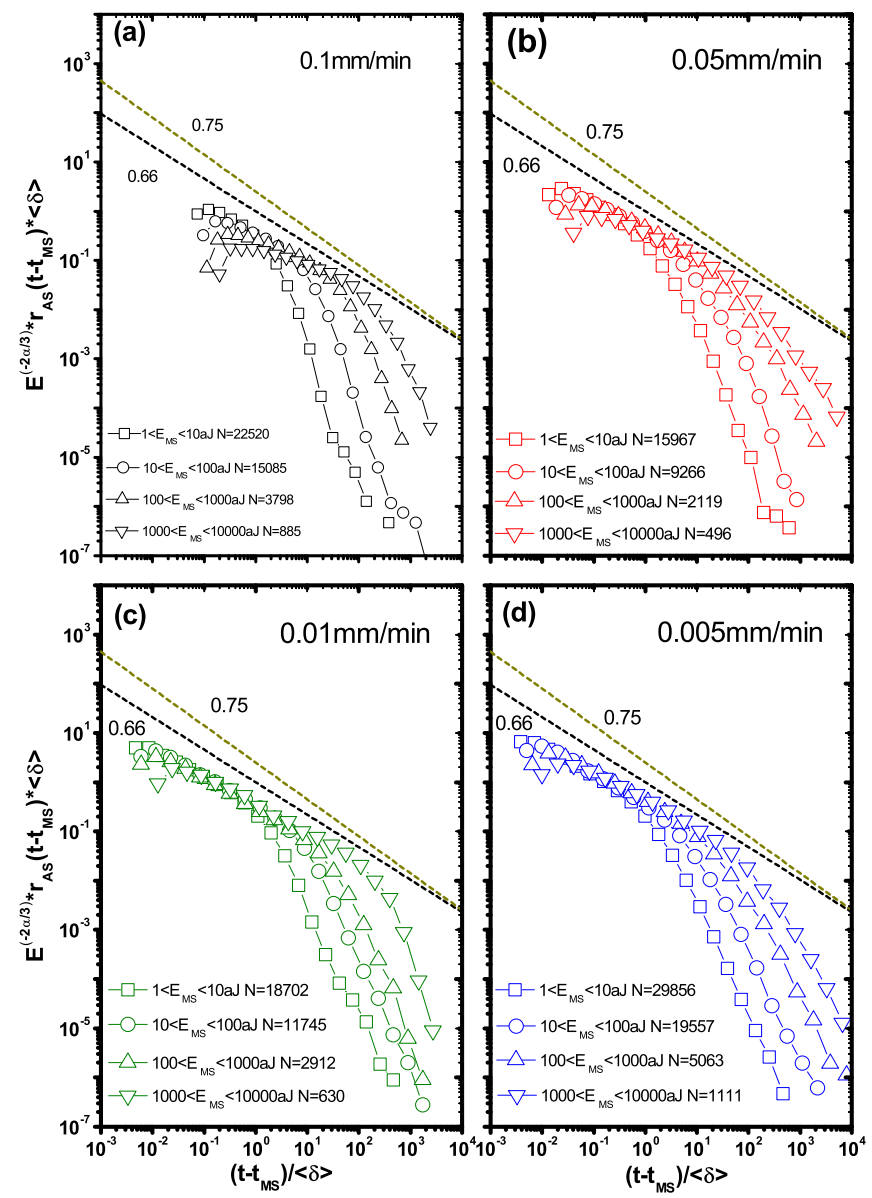

FIG. 5. Aftershock rate as a function of the temporal distance to the mainshock in log-log scales and logarithmic bins for charcoal samples. According to the productivity law, data have been scaled by the energy of the mainshock to the power $-2 \alpha / 3$ with $\alpha=0.5$. The lines indicate the Omori exponents $p=0.75$ and $p=0.66$. The different symbols correspond to the analysis of the mainshocks in different energy windows, as indicated by the legend. $N$ indicates the number of mainshocks in each window. The four panels correspond to different compression rates as indicated.

scaling laws in log-log plots. As explained in the previous section, the plots show a double power-law behavior with exponents $(1-v)=0.93$ and $(2+\xi)=2.45$. These values, found previously for Vycor experiments [8], are indicated with straight lines. Only when the compression rate is too fast $[0.1 \mathrm{~mm} / \mathrm{min}$, Fig. $4(\mathrm{a})]$, the behavior corresponding to small waiting times seems to exhibit a smaller exponent. This can be understood since, at fast compression rates, large avalanches overlap with the aftershocks, destroying the short time attractive correlations and thus giving a behavior which is more Poissonian.

Figure 5 shows the analysis of the aftershock rate for the charcoal specimens at different compression rates. The data are also scaled according to the productivity law (5) as explained in Sec. II. The best $\alpha$ exponent for the scaling has been found to be $\alpha=0.5$. As expected when the energy window for the definition of $M S$ is high enough the plot shows an Omori behavior with an exponent $p \simeq 0.66$. This value is 

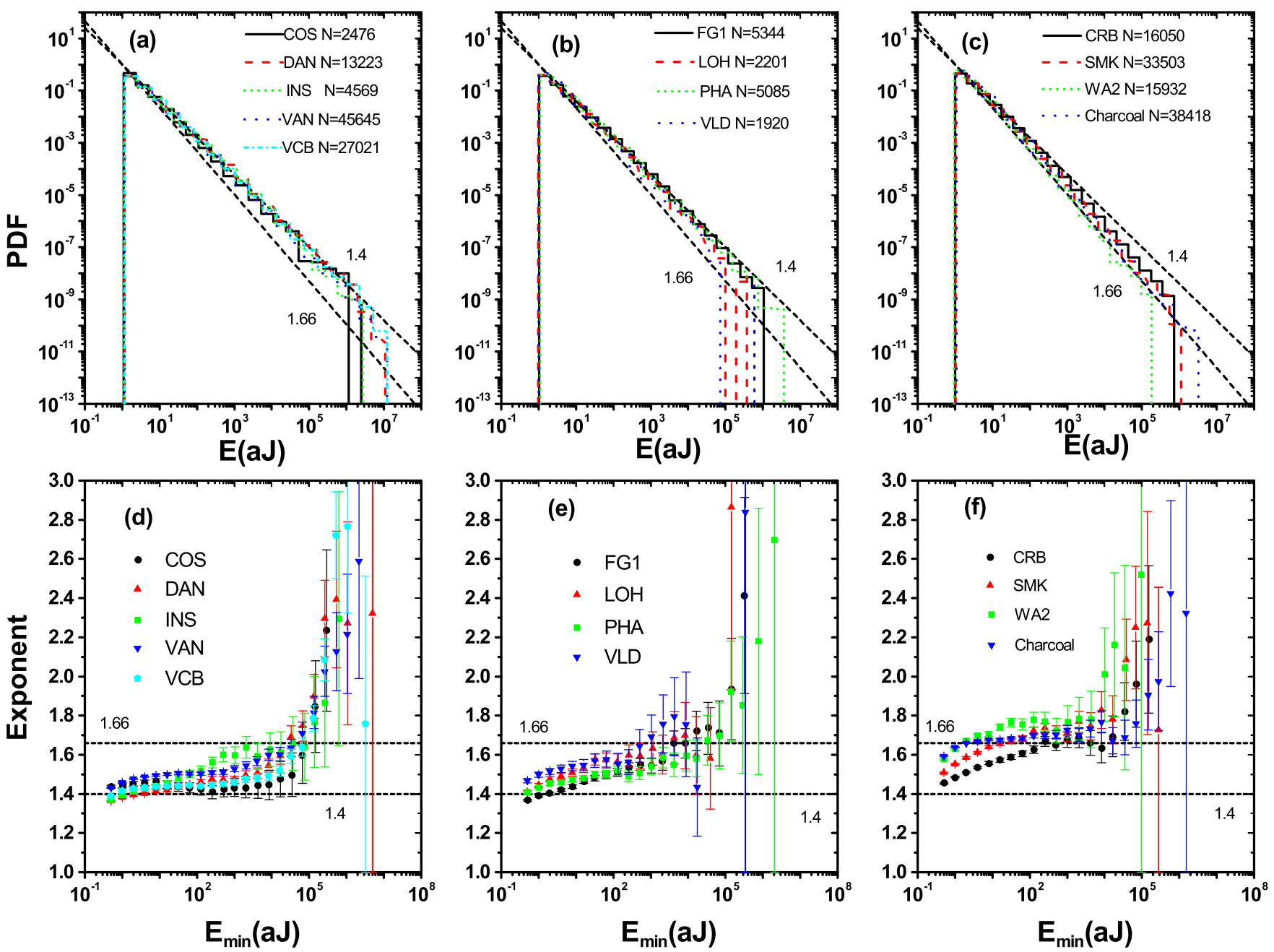

FIG. 6. Gutenberg-Richter energy distributions for coal samples in groups I (a), II (b), and III (c), in log-log scales and with logarithmic bins. The dashed lines show the behaviours corresponding to $\epsilon=1.66$ and $\epsilon=1.44$ as indicated. The plots below show the exponents fitted by the ML method a a function of a lower cutoff $E_{\min }$. The dahsed hortizontal lines also correspond to the exponents $\epsilon=1.66$ and $\epsilon=1.44$.

different from the value found for Vycor [8], as can be seen by comparing with the dashed lines.

The results presented so far, already show that charcoal samples exhibit a rather good critical behavior characterized by critical exponents which, in some cases $(\epsilon$ and $p)$ differ from the ones found in Vycor experiments. The value of $\epsilon$, nevertheless, is much closer to the value found for earthquakes. The critical behavior is rather robust, extending almost five decades in the Gutenberg-Richter distribution and rather independent of the compression rate.

In the next set of experiments we systematically analyze the specimens corresponding to the natural coal samples. As will be shown samples can be separated in three different groups with different sets of exponents. Group I that contains the specimens from the COS, DAN, INS, VAN, and VCB samples exhibit exponents and statistical properties very similar to those found previously for Vycor [8]; group III containing CRB, SMK, and WA2 samples exhibits properties similar to those of charcoal, presented above; and group II containing FG1, LOH, PHA, and VLD exhibit a mixed behavior.

Figure 6 shows the AE energy distributions for the three groups (top panels) and the corresponding ML analysis of the $\epsilon$ exponent as a function of the cutoff $E_{\min }$ (bottom panels). The behaviors corresponding to Vycor $(\epsilon=1.4)$ and charcoal $(\epsilon=1.66)$ are indicated by dashed lines. It is clear that the samples in the first group show a rather good powerlaw behavior, corroborated by the almost constant plateau in the ML analysis, compatible with the Vycor exponent. The sample INS is the only one with a less constant plateau but it has been included in this group because the fitted $\epsilon$ exponent is smaller than 1.66 for values of $E_{\min }$ up to $10^{3}$ aJ. Contrarily, samples in group III show a much steeper histogram with a slope clearly higher than 1.4. The data for charcoal are also included in the plots corresponding to group III for comparison. The ML analysis reveals that already for cutoffs $E_{\min }$ above $10^{2}$ aJ, the exponent is already compatible with $\epsilon=1.66$, the value found for charcoal in the previous analysis. Nevertheless, it should be mentioned that none of the CRB, SMK, and WA2 samples shows a very flat plateau as the one exhibited by charcoal samples. Finally, group II samples show a histogram that apparently seems to follow the $\epsilon=1.4$ slope, but the ML analysis reveals that the fitted effective exponent increases monotonously from 1.4 to values above 1.66 for $E_{\min }>10^{3}$ aJ. This behavior could be explained 

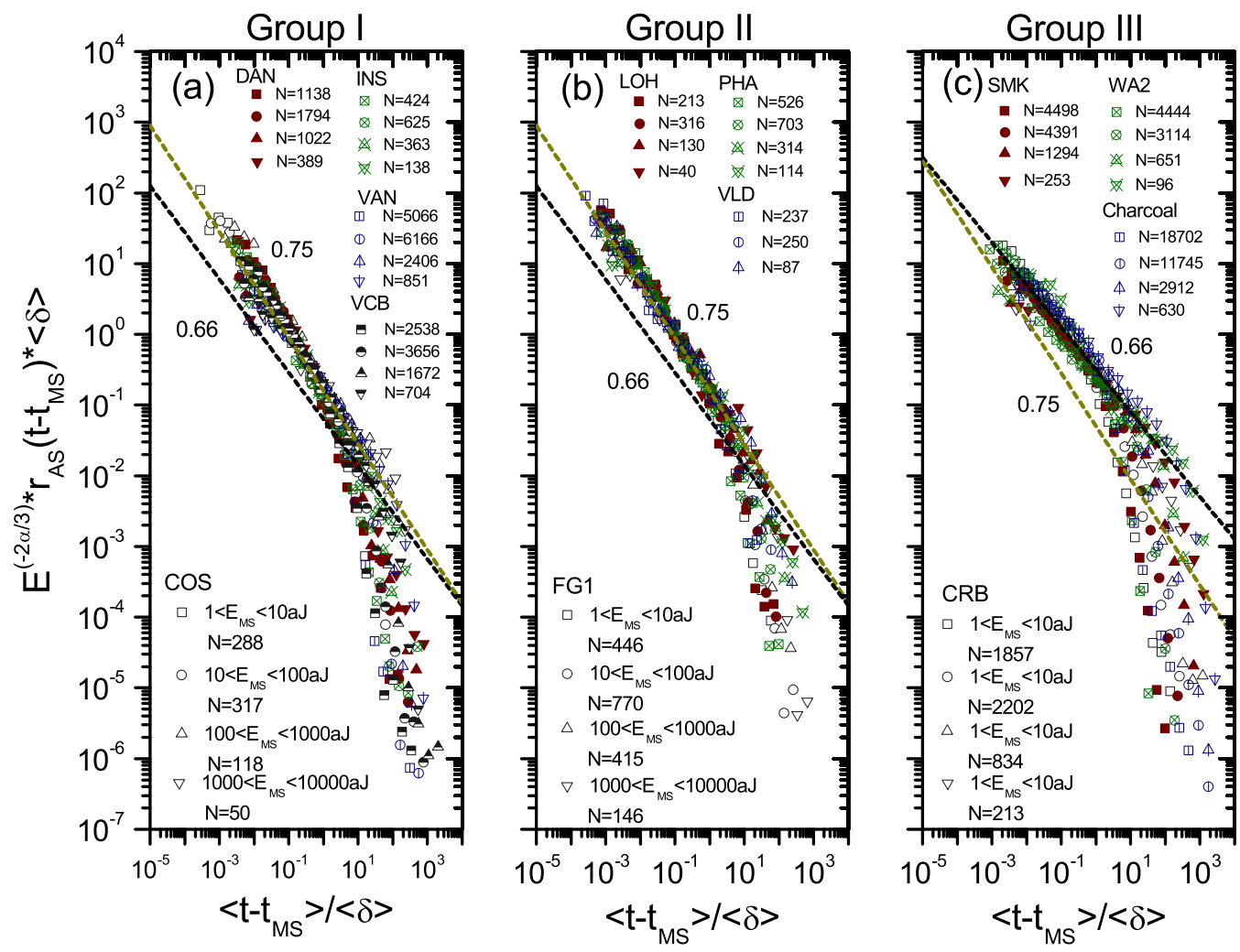

FIG. 7. Scaling of the rate of aftershocks for the tree different groups. In all cases we used $\alpha=0.5$. The dashed lines indicate the values $p=0.4$ and $p=0.66$.

[26] assuming that theses samples show a certain exponential factor with a characteristic energy that multiplies the $\epsilon=1.4$ power-law distribution, as described by Eq. (2).

Figure 7 shows the analysis of the rate of aftershocks, scaled according to Eq. (5) for the three groups of samples. On each plot, different colors correspond to different samples and different symbols to different energy windows for the definition of the mainshocks. The width of the windows is indicated in the labels. The $N$ values indicate the number of events in every set. Scaling has been obtained in all cases with a productivity exponent $\alpha=0.5$. The slope in log-log plots corresponds to $p=0.75$ for groups I and II and $p=0.66$ for group III as indicated by the dashed lines. The samples in group III exhibit a clear lower Omori exponent $p$, thus a larger persistence of the AS sequences,

Figure 8 shows the analysis of the distribution of waiting times for the three groups of samples. As can be seen, in this case, the three groups show a very good collapse corresponding to the Universal Scaling Law (USL) with two power-law tails at large and small waiting times. The collapses include different samples and analysis corresponding to different energy thresholds. For all cases the behavior at large and short waiting times can be described with the same critical exponents $(1-v)=0.93$ and $(2+\xi)=2.45$, in agreement with the values found previously for Vycor [8]. Thus, these exponents $[(1-v)$ and $(2+\xi)]$ are clearly more robust (universal) than the $\epsilon$ and $p$ exponents that change from group to group.

The results are summarized in Table II. The exponents obtained from Group II are not included since they are equal to those corresponding to Group I. The only difference is that, for Group II, the Gutenberg-Richter energy distribution shows an effective exponent $\epsilon=1.4$ that increases when the small energy data is thresholded due to, most probably, the existence of an exponential damping factor.

It should also be recalled that the $\epsilon$ exponent typically extends for more decades for samples in Group I. For Group III samples the plateaux of the ML analysis are not as long and extend for two decades or less.

\section{DISCUSSION}

After the phenomenological identification of the groups with rather equivalent statistical properties and time correlations, the remaining question is to identify if there are other physical properties that would reinforce such a separation.

When looking at Table I one can see that samples DAN, VCB, and VAN, corresponding to Group I, are located at the bottom. This corresponds to highly evolved coals for which the different macerals have already rather similar chemical and physical properties. The two exceptions are INS and COS. These samples are much younger in rank, but as can be seen, they are also very homogeneous since they are formed essentially by vitrinite and all of them have very low ash yields. Thus physicochemical homogeneity would be a common feature for samples in Group I. The example micrographs INS and DAN shown in Fig. 1 also support the homogeneity of such samples. Another common point would be that porosity in these highly evolved coals (or vitrinite rich coals) is mostly in the micro and mesoscale $(<50 \mathrm{~nm})$. 

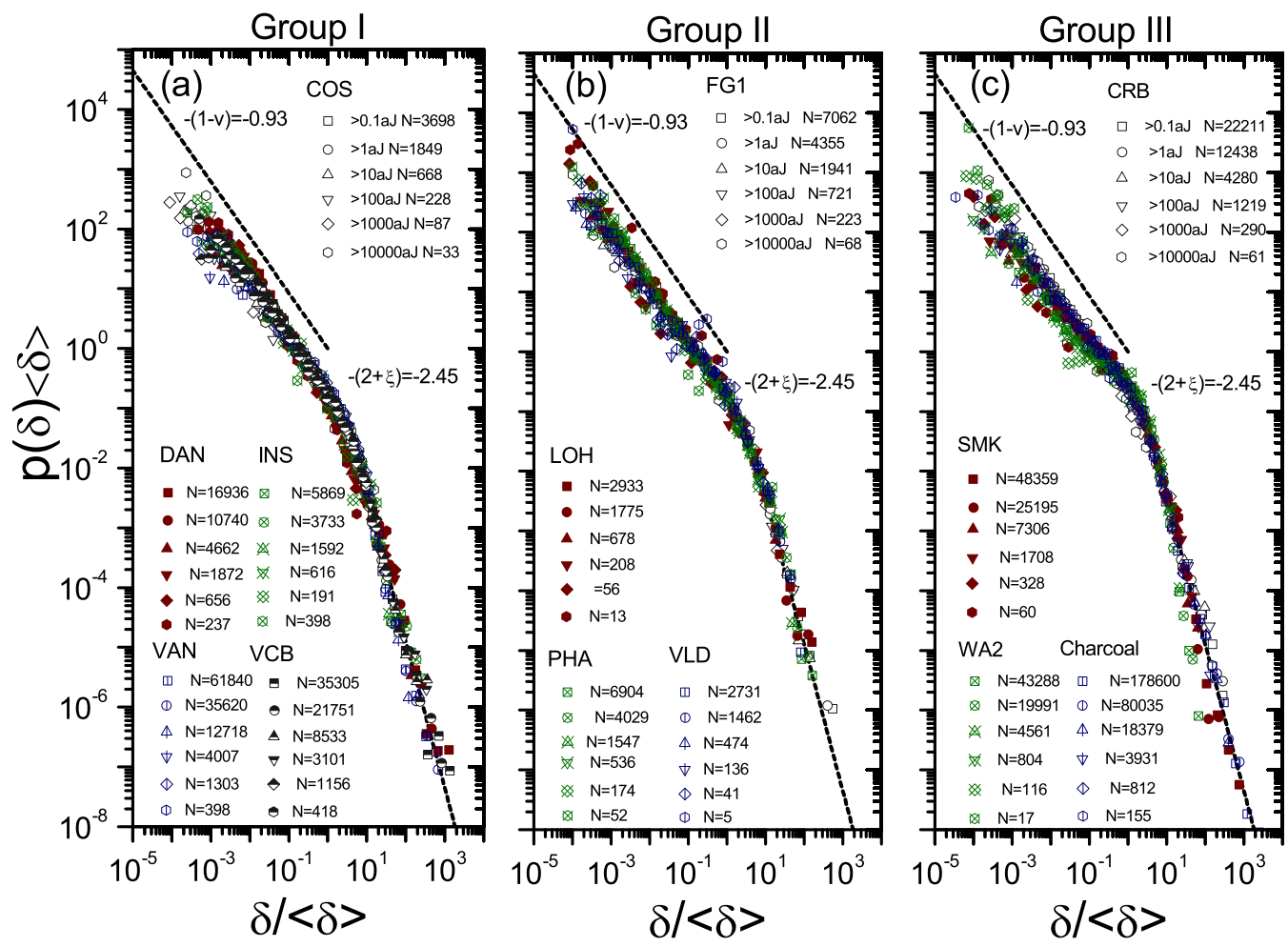

FIG. 8. Distribution of waiting times, scaled according to the Universal Saclaing Law, for the three groups of samples. The dashed lines indicate the exponents $(1-v)=0.93$ and $(2+\xi)=2.45$.

Samples SMK, CRB, and WA2, in Group III, contrarily are samples with a significant inertinite content which is close to the amount of vitrinite (see Table I). Inertinite has a carbon skeleton more disordered than vitrinite with predominance of $\mathrm{C}-\mathrm{C}$ covalent bonds arranged in a three-dimensional (3D) net with greater porosity than vitrinite and with lower $\mathrm{H}$ content [35]. These characteristics are also common to charcoal that can be considered an artificially prepared inertinite, which plot in the same Group III. Micrographs of charcoal, WA2, and CRB in Fig. 1 also confirm the high heterogeneous character of samples in this group. They also share the existence of voids and/or macropores ( $>50 \mathrm{~nm}$ )

Moreover, we can increase our understanding about the different mechanical behavior under compression by plotting examples of the stress-strain curves of specimens in the three groups. These are shown in Figs. 9(a)-9(c). Different colors correspond to different samples. The vertical axis, corresponding to the measured force, has been normalized by the maximum value of the stress (yield stress). Although there is a large variability in such curves, one can appreciate some qualitative differences, specially between samples in Groups I and III.

The typical signature of samples in Group I is that stress drops associated with the sample failures are rather sharp and followed, in most cases, by a subsequent almost linear (elastic) increase of the force. In many cases, this elastic increase of the force reaches values well above the force before the drop. This indicates the existence of hardening effects during most of the compression process. Besides, in this Group I, most of the experiments terminate with a large failure involving the full sample, as in perfectly brittle or quasibrittle samples, after which the force falls below negligible values.

For Group III, contrarily, most of the force drops are not so sharp and typically involve several consecutive decreasing steps. After most of the drops, the curve follows flat (like in dynamic friction experiments) or decreasing trajectories.

TABLE II. Comparsion of the exponents found in this work for Charcoal and coal samples in Groups I and III. Data are compared to previous exponents found for Vycor. We also include some of the values (or range of values) proposed for earthquakes, although there is a large variability and controversy in the bibliography. For a discussion of the exponent $\epsilon$ see Ref. [23]; for exponents $p$ and $\alpha$ see Ref. [28]; and for exponents $(1-v)$ and $(2+\xi)$, see Ref. [29].

\begin{tabular}{lcccccc}
\hline \hline & $\epsilon$ & $p$ & $\alpha$ & $(1-v)$ & $(2+\xi)$ & Ref. \\
\hline Vycor & $1.4 \pm 0.05$ & $0.75 \pm 0.1$ & $0.5 \pm 0.1$ & $0.93 \pm 0.05$ & $2.45 \pm 0.08$ & {$[8]$} \\
coal group I & 1.4 & 0.75 & 0.5 & $0.93 \pm 0.05$ & $2.45 \pm 0.08$ & this work \\
Charcoal & 1.66 & 0.66 & 0.5 & $0.93 \pm 0.05$ & $2.45 \pm 0.08$ & this work \\
coal group III & 1.66 & 0.66 & 0.5 & $0.93 \pm 0.05$ & $2.45 \pm 0.08$ & this work \\
Earthquakes & $1.66(1.33-2.33)$ & $(0.9-1.0)$ & $0.8(0.2-1.9)$ & $0.9(0.5-0.95)$ & 2.20 & {$[23,28,29]$} \\
\hline \hline
\end{tabular}



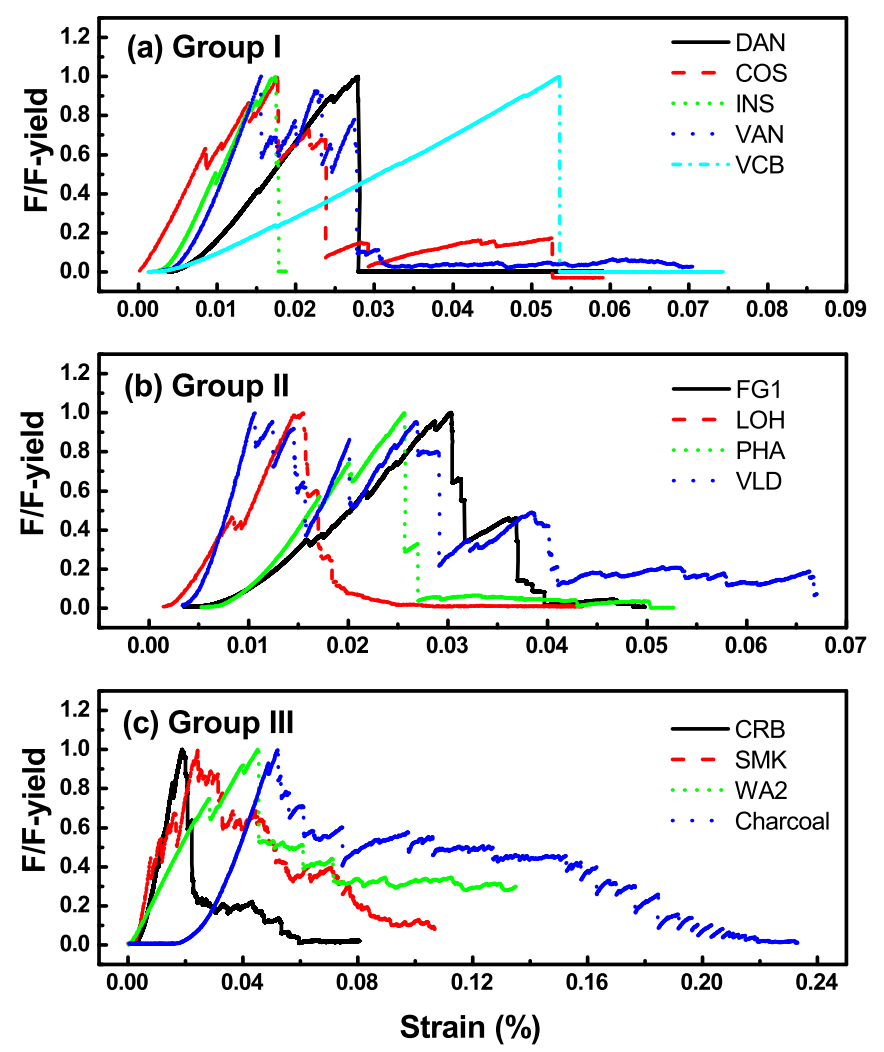

FIG. 9. Examples of stress strain curves during the compression experiments of samples in the three groups. Different lines correspond to different samples as indicated. The vertical axis has been normalized by the maximum force (yield point). Note that the horizontal scale in (c) is much larger than in (a) and (b).

In the case that the trajectories increase after the drops, they clearly show a curvature indicative of effective plasticity. This points towards the existence of weakening mechanisms during most of the compression process. Moreover, the failure after the yield point is not abrupt. It needs a longer deformation during which a lot of acoustic activity occurs. In general, these samples admit a much larger deformation after the yield point, as can be seen by noting that the horizontal scale in Fig. 9(c) is three times longer than Figs. 9(a) and 9(b).

Therefore, our experimental evidences reveal that the more homogeneous, less porous samples in Group I ( $\epsilon=1.4$ and $p=0.75)$ tend to exhibit more hardening effects while the more heterogeneous and porous samples in Group III $(\epsilon=$ 1.66 and $p=0.66$ ) exhibit more weakening effects.

The results in this work can be compared to previous experimental data. Compression experiments of wood samples [15] have given an exponent $\epsilon=1.37$ for constant velocity (and $\epsilon=1.4$ for force driven experiments). They also show $p=$ $0.75, \alpha=0.4,(1-v) \simeq 1$, and $(2+\xi)>2$. These results are totally compatible with the data obtained from Vycor and samples in Group I. The stress-strain curves also indicate that fracture events mostly occur during an average increasing curve thus revealing a certain degree of hardening. Recently the fact that Vycor does not display a unique critical failure point (that will correspond to a brittle fracture) but a series of periods of accelerated seismic release has also been attributed to the existence of hardening effects [21].

An experimental study of $\mathrm{SiO}_{2}$ materials compared several Gelsil synthetic samples and natural sandstone [10] with previous Vycor data. The composition of these materials is essentially the same $\left(\mathrm{SiO}_{2}\right)$ as Vycor. The difference is that Vycor has a porous structure at the micro and nanoscale with a very homogeneous matrix obtained by a phase separation and leaching process, Gelsil has a similar porosity and purity but is obtained by a sol-gel hydrolization process and high temperature treatment of quatrz powders. Natural sandstone is much more heterogeneous, contains many impurities and shows a granular structure, in some cases with low adhesion between grains. It was found that the $\epsilon$ exponent for Gelsil was essentially the same than for Vycor $\epsilon=1.35-1.4$ but for the different sandstone samples the exponent showed larger effective values $\epsilon \sim 1.44-1.55$. The ML analysis revealed for these samples a slope that could be indicative of the existence of damping factors like we found for coal samples of Group II in the present work. Concerning the exponent governing the temporal correlations $p$ values where rather similar for all the samples in the range $p \sim 0.70-0.78$, also in agreement with Group II results, but the $\alpha$ exponents were larger for natural sandstone (up to $\alpha=0.7-0.8$ ). The exponents of the Universal Scaling Law were also relatively similar to the ones found in all the samples in the present work with values $(1-v) \sim 0.9-1.1$. and $(2+\xi) \sim 1.4-3.0$.

Very recently, a study of AE under compression of manganite samples with different microstructures (obtained by different synthesis methods) [20] revealed that the statistical distribution of energies is characterized by a power-law exponent $\epsilon=1.45$ for samples with large grains but shows damping factors and larger effective exponents when the grains are smaller. The change has been associated with two different mechanisms: large grain fracture in the first case and grain friction in the second.

Simulation studies of compression of a porous sedimentary rock based on randomly sized spherical particles [40] render an exponent for the energy distribution of avalanches $\epsilon=$ 2.02 , with a clear damping factor. This is a value larger than the two values that we found. We cannot provide an explanation for this discrepancy. Mean-field micromechanical models for deformation in solids render the same exponent $\tau=1.5$ for the size distribution of avalanches for both hardening and brittle cases [41]. The comparison of this exponent with our $\epsilon$ values is not straightforward because it will require an assumption about the relation of the avalanche energies and sizes that, to our knowledge, is at present not well established.

\section{CONCLUSION}

In summary, from the systematic study of coal and charcoal samples we can propose the following scenario. Failure under uniaxial compression of porous materials follows two different mechanisms that, eventually, can lead to the classification of the systems into two different classes.

(a) For the more homogeneous samples (samples in Group I), without voids and macropores and with less granular microstructures, the compression process is characterized by a sequence of failure events followed by hardening trajecto- 
ries. The associated acoustic emission energies are power-law distributed with an exponent $\epsilon \sim 1.4$. The process, in these materials, terminates suddenly in a brittle-like event in which a large fraction of the sample collapses. These samples show almost identical exponent values than those found previously for Vycor, that would be the paradigmatic example for this class.

(b) For the more heterogeneous samples (samples in Group III), with voids or macropores, with granular like structures due to the existence of two or more macerals with clear different physical properties, the compression process exhibits weakening behavior and dynamic friction like behavior. The associated AE energies are also power-law distributed with an exponent $\epsilon \sim 1.66$, although in this case the distributions show less decades of power-law behavior and are often distorted by exponential damping factors. Charcoal would be the best example in this class showing a very good powerlaw behavior, extending approximately five decades. The $\epsilon$ exponent obtained in this case is in better agreement with the Gutenberg-Richter exponent found in seismology $\epsilon \sim$ $5 / 3 \sim 1.66$. Thus Earth's crust behavior is suggested to fall into this second class. Indeed its compositional and structural heterogeneity is known to prevail over a wide range of scales [42]. It also shows clear signatures of weakening effects [43] due to frictional heating and fault gouge.

The samples in Group II, show intermediate acoustic and mechanical behavior between the other two groups. It is difficult to really describe them as a different class, but most probably as samples exhibiting crossover from homogeneous to heterogeneous. Their energy distributions can be understood as damped power laws with an exponent (for low energies) similar to homogeneous samples in Group I. The damping would be caused by the existence of some characteristic scales: grains, inhomogeneities, macropores, and so on.

Concerning time correlations all samples show aftershock clustering correlations (Omori-like). For the heterogeneous samples the power-law decay is weaker, thus aftershocks occur with more frequency for relatively longer times than for the homogeneous samples. The fact that the Omori exponents in our experiments do not correspond to the exponents observed in seismology could be related to the fact that we cannot experimentally locate the origin of the AE events and therefore we cannot take into account the information about distances for the correct identification of aftershocks. This will require more experimental efforts to locate the position of the labquake sources.

An important conclusion is that the so-called Universal Scaling Law for waiting times distributions seems to be common to all our studied samples with exponents very similar to those found in seismological large catalogues. This suggests that the reasons behind the numerical values of the exponents $(1-v)$ and $(2+\xi)$ shall be rather fundamental, independent of the sample properties and length scales, related to general properties of stochastic point processes at criticality.

\section{ACKNOWLEDGMENTS}

Y.X. and X.D. acknowledge financial support from National Natural Science Foundation of China (Grants No. 51571156, No. 51671157, No. 51621063, and No. 51320105014). E.V. and A.P. acknowledge financial support from the Spanish Ministry of Economy and Competitiveness (MAT2016-75823-R). We also acknowledge Dr. Jordi Baró for fruitful discussions.
[1] J. P. Sethna, K. A. Dahmen, and C. R. Myers, Crackling noise, Nature 410, 242 (2001).

[2] E. Vives, J. Ortín, L. Mañosa, I. Ràfols, R. Pérez-Magrané, and A. Planes, Distributions of Avalanches in Martensitic Transformations, Phys. Rev. Lett. 72, 1694 (1994).

[3] S. Zapperi, A. Vespignani and H. E. Stanley, Plasticity and

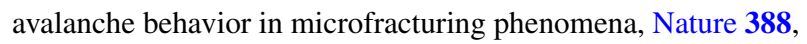
658 (1997).

[4] D. Spasojević, S. Bukvić, S. Milošević, and H. E. Stanley, Barkhausen noise: Elementary signals, power laws, and scaling relations, Phys. Rev. E 54, 2531 (1996).

[5] E. K. H. Salje, E. Dul'kin, and M. Roth, Acoustic emission during the ferroelectric transition $\mathrm{Pm} \overline{3} \mathrm{~m}$ to $\mathrm{P}_{4} \mathrm{~mm}$ in $\mathrm{BaTiO}_{3}$ and the ferroelastic transition $\mathrm{R} \overline{3} \mathrm{~m}-\mathrm{C} 2 / \mathrm{c}$ in $\mathrm{Pb}_{3}\left(\mathrm{PO}_{4}\right)_{2}$, Appl. Phys. Lett. 106, 152903 (2015).

[6] W. Wu and P. W. Adams, Avalanches and Slow Relaxation: Dynamics of Ultrathin Granular Superconducting Films in a Parallel Magnetic Field, Phys. Rev. Lett. 74, 610 (1995).

[7] M. P. Lilly, P. T. Finley, and R. B. Hallock, Memory, Congruence, and Avalanche Events in Hysteretic Capillary Condensation, Phys. Rev. Lett. 71, 4186 (1993).

[8] J. Baró, A. Corral, X. Illa, A. Planes, E. K. H. Salje, W. Schranz, D. E. Soto-Parra, and E. Vives, Statistical Similarity between the Compression of a Porous Material and Earthquakes, Phys. Rev. Lett. 110, 088702 (2013).
[9] V. Navas-Portella, I. Serra, A. Corral, and E. Vives, Increasing power-law range in avalanche amplitude and energy distributions, Phys. Rev. E 97, 022134 (2018).

[10] G. F. Nataf, P. O. Castillo-Villa, J. Baró, X. Illa, E. Vives, A. Planes, and E. K. H. Salje, Avalanches in compressed porous $\mathrm{SiO}_{2}$-based materials, Phys Rev. E 90, 022405 (2014).

[11] P. O. Castillo-Villa, J. Baró, A. Planes, E. K. H. Salje, P. Sellappan, W. M. Kriven, and E. Vives, Crackling noise during failure of alumina under compression: the effect of porosity, J. Phys.: Condens. Matt. 25, 292202 (2013).

[12] G. F. Nataf, P. O. Castillo-Villa, P. Sellappan, W. M. Kriven, E. Vives, A. Planes, and E. K. H. Salje, Predicting failure: acoustic emission of berlinite under compression, J. Phys.: Condens. Matt. 26, 275401 (2014).

[13] E. K. H. Salje, G. I. Lamprosi, D. E. Soto-Parra, J. Baró, A. Planes, and E. Vives, Noise of collapsing minerals: Predictability of the compressional failure in goethite mines, American Mineralogist 98, 609 (2013).

[14] J. Baró, A. Planes, E. K. H. Salje, and E. Vives, Fracking and labquakes, Phil. Mag. 96, 3686 (2016).

[15] T. Mäkinen, A. Miksic, M. Ovaska, and M. J. Alava, Avalanches in Wood Compression, Phys. Rev. Lett. 115, 055501 (2015).

[16] J. Baró, P. Shyu, S. Pang, I. M. Jasiuk, E. Vives, E. K. H. Salje, and A. Planes, Avalanche criticality during compression 
of porcine cortical bone of different ages, Phys. Rev. E 93, 053001 (2016)

[17] H. V. Ribeiro, L. S. Costa, L. G. A. Alves, P. A. Santoro, S. Picoli, E. K. Lenzi, and R. S. Mendes, Analogies Between the Cracking Noise of Ethanol-Dampened Charcoal and Earthquakes, Phys. Rev. Lett. 115, 025503 (2015).

[18] X. Jiang, H. Liu, I. G. Main, and E. K. H. Salje, Predicting mining collapse: superjerks and the appearance of record-breaking events in coal as collapse precursors, Phys. Rev. E 96, 023004 (2017).

[19] V. Navas-Portella, A. Corral, and E. Vives, Avalanches and force drops in displacement-driven compression of porous glasses, Phys. Rev. E 94, 033005 (2016).

[20] D. Soto-Parra, E. Vives, M. E. Botello-Zubiate, J. A. MatutesAquino, and A. Planes, Acoustic emission avalanches during compression of granular manganites, Appl. Phys. Lett. 112, 251906 (2018).

[21] J. Baró, K. A. Dahmen, J. Davidsen, A. Planes, P. O. Castillo, G. F. Nataf, E. K. H. Salje, and E. Vives, Experimental Evidence of Accelerated Seismic Release without Critical Failure in Acoustic Emissions of Compressed Nanoporous Materials, Phys. Rev. Lett. 120, 245501 (2018).

[22] T. C. Hanks and H. Kanamori, A moment magnitude scale, J. Geophys. Res. 84, 2348 (1979).

[23] Y. Kagan, The universlaity of the frequency-magnitude relationship, Pure Appl. Geophys. 155, 537 (1999).

[24] H. Bauke, Parameter estimation for power-law distributions by maximum likelihood methods, Eur. Phys. J. B 58, 167 (2007).

[25] A. Clauset, C. Rohilla-Shalizi, and M. E. J. Newman, Powerlaw distributions in empirical data, SIAM Rev. 51, 661 (2009).

[26] E. K. H. Salje, A. Planes, and E. Vives, Analysis of crackling noise using the maximum-likelihood method: Power-law mixing and exponential damping, Phys. Rev. E 96, 042122 (2017).

[27] T. Utsu, Y. Ogata, and R. S. Matsu'ura, The centenary of the omori formula for a decay law of aftershock activity, J. Phys. Earth 43, 1 (1995).

[28] A. Helmstetter, Is Earthquake Triggering Driven by Small Earthquakes? Phys. Rev. Lett. 91, 058501 (2003).

[29] A. Corral, Universal local versus unified global scaling laws in the statistics of seismicity, Physica A 340, 590 (2004).
[30] A. Helmstetter and D. Sornette, Subcritical and supercritical regimes in epidemic models of earthquake aftershocks, J. Geophys. Res. 107, ESE 10-1 (2002).

[31] E. Stach, M.-Th. Mackowsky, M. Teichmüller, G. H. Taylor, D. Chandra, and R. Teichmüller, Stach's Textbook of Coal Petrology (Gebrüder Borntraeger, Berlin, 1982).

[32] M. Lindman, K. Jonsdottir, R. Roberts, B. Lund, and R. Bödvarsson, Earthquakes Descaled: On Waiting Time Distributions and Scaling Laws, Phys. Rev. Lett. 94, 108501 (2005).

[33] G. H. Taylor, M. Teichmüller, A. Davis, C. F. K. Diessel, R. Littke, and P. Robert, Organic Petrology (Gebrüder Borntraeger, Berlin, 1998).

[34] C. F. K. Diessel, Coal and Coal-Bearing Depositional Systems (Springer-Verlag, Berlin, 1992).

[35] D. van Krevelen, Coal (Elsevier, Amsterdam, 1993).

[36] A. G. Borrego, G. Marbán, M. J. G. Alonso, D. Alvarez, and R. Menéndez, Maceral effects in the determination of proximate volatiles in coal, Energy Fuels 14, 117 (2000).

[37] A. Scott and I. Glasspool, Observations and experiments on the origin and formation of inertinite group macerals, Int. J. Coal Geol. 70, 53 (2007).

[38] M. A. Diez and H. Marsh, in Sciences of Carbon Materials, edited by H. Marsh and F. Rodnguez-Reinoso (Publicaciones de la Universidad de Alicante, Alicante, Spain, 2000), pp. 571-593.

[39] F. J. Pérez-Reche, B. Tadiç, L. Manosa, A. Planes, and E. Vives, Driving Rate Effects in Avalanche Mediated First-Order Phase Transitiions, Phys. Rev. Lett 93, 195701 (2004).

[40] F. Kun, I. Varga, S. Lennartz-Sassinek, and I. G. Main, Rupture Cascades in a Discrete Element Model of a Porous Sedimentary Rock, Phys. Rev. Lett. 112, 065501 (2014).

[41] K. A. Dahmen, Y. Ben-Zion, and J. T. Uhl, Micromechanical Model for Deformation in Solids with Universal Predictions for Stress-Strain Curves and Slip Avalanches, Phys. Rev. Lett. 102, 175501 (2009).

[42] See, for instance, some of the papers in Heterogeneity in the Crust and Upper Mantle: Nature, Scaling, and Seismic Properties, edited by J. A. Goff and K. Holliger, (Springer, New York, 2003).

[43] J. R. Rice, Heating, weakening and shear localization in earthquake rupture, Philos. Trans. A Math. Phys. Eng. Sci. 375, 20160015 (2017). 